\title{
A Comprehensive Review on the Working of a Radiation Oncology Facility During the Covid-19 Pandemic and Adapting it for South Asian Settings
}

\author{
Kunhi Parambath Haresh ${ }^{1}$, Yousra Izzudeen ${ }^{1}$, Anil Gupta ${ }^{1}$, Narendra Kumar, ${ }^{2,3}$, \\ Devangana Bora ${ }^{1}$, Jagadesan Pandjatcharam ${ }^{4}$, Amit Bahl' ${ }^{2}$, Umesh Velu ${ }^{5}$, Subhas \\ Pandit $^{6}$, Krishnanunni Vijayakumar Ajitha ${ }^{1}$,Khan Mohammad Masud Rana ${ }^{3}$, \\ Lakshman Obeysekara ${ }^{7}$, Subhash Gupta ${ }^{1}$, Goura Kishor Rath ${ }^{1,8}$
}

${ }^{1}$ Department of Radiotherapy, AIIMS, New Delhi, India. ${ }^{2}$ PGIMER, Chandigarh, India. ${ }^{3}$ Evercare Hospital, Dhaka, Bangladesh. ${ }^{4} \mathrm{JIPMER}$, Pondicherry, India. ${ }^{5}$ Kasturba Medical College, Manipal, India. ${ }^{6}$ Kathmandu Cancer Hospital, Nepal. ${ }^{7}$ Apeksha National Cancer Center, SriLanka. ${ }^{8} \mathrm{NCI}-\mathrm{AIIMS}$, Jhajjar, Haryana, India.

\begin{abstract}
Background: With the pandemic gaining a firm foothold globally, various governments world-wide are trying hard to halt its unprecedented spread. The pandemic is challenging the healthcare professionals in unique ways and forcing the frontline fighters to come up with dynamic changes in almost all disciplines of medical science. This article is aimed at a detailed review of the existing guidelines for radiotherapy practice during this pandemic from across the world. Methods: This review has been organised under specific subheadings that pertains to the functioning of a Radiation Oncology facility in South Asian countries like India. After a detailed Zoom video conference between the authors, it was decided to focus the review under the following subheadings: staff allocation, staff education, screening of patients, patient waiting area modification, patient selection, radiotherapy planning and execution, review of patients on radiotherapy, brachytherapy, inpatient admissions, follow up, resident training and treatment of suspected or positive COVID-19 patients. Results: After discussion among the authors, a consensus working suggestion during the COVID-19 pandemic has been proposed for a radiotherapy center in a South Asian country like India. All the authors worked simultaneously on a Google doc document to develop this manuscript. Conclusions: This paper can be a reference document for the functioning of a radiotherapy facility during the COVID19 pandemic. As the infrastructure of different institutes vary and so does each patient, the importance of fine tuning and tailoring our final decisions before treating a patient in this unprecedented crisis cannot be undermined.
\end{abstract}

Keywords: COVID 19- Pandemic- Radiotherapy- External Beam- Brachytherapy

\section{Introduction}

Ever since the first case of Novel Coronavirus was reported from China, by the World Health Organization (WHO) on 31st of December 2019, it has spread to various countries. The biggest impact has been on the healthcare system. In India the first case was reported in a student from Kerala who had returned from Wuhan on the $30^{\text {th }}$ of January, 2020. India reported its first death on $12^{\text {th }}$ March in a 76 year old man who had returned from Saudi Arabia. Despite stringent measures, there is a linear rise in the number of infections. Not only are cancer patients more susceptible to COVID-19, they also are at a higher risk of suffering from severe events like ICU admissions

Corresponding Author:

Dr. Yousra Izzuddeen

Department of Radiotherapy, AIIMS, New Delhi, India.

Email: yustmc@gmail.com 
requiring ventilation, or death [1]. The world had already seen the catastrophic consequences that the pandemic had on countries which are known for their premier healthcare delivery systems like Italy, the United States and the United Kingdom. It is imperative that we learn from their experiences. These advisories and contingency plans are intended to cover in depth the nuances of running a radiation oncology department in South-Asian countries.

\section{Materials and Methods}

The authors did a thorough literature search in PUBMED and Google looking for published articles relating to COVID-19 and Radiotherapy. The websites of all important oncology Journals, directives from the Oncology societies and different institutional guidelines were also searched. The authors conducted video conferencing meetings using the Zoom App and the broad headings for the article being written was agreed upon. The manuscript was worked on google docs simultaneously by the authors from their respective places. Our approach of teleconferencing and shared document platforms is all the more important at this juncture when physical distancing is the new norm. At the time of writing this paper there were 38 publications, 6 society guidelines and 10 institutional guidelines available. This review will be covered under 13 sub headings:

\section{Results and Discussion}

\section{Staff Allocation}

Published guidelines: Radiotherapy centres in Wuhan had created an ad-hoc emergency infection control team responsible for activities related to control of infection [2]. Staff members comprising Radiation Oncologists, Radiation Therapists and nurses were divided into groups of $\mathrm{A}$ and $\mathrm{B}$, each working for a specified number of days followed by 7-10 days break, the estimated incubation period, to avoid exposure simultaneously [2]. Experience from Singapore highlighted the importance of segregation of staff even at work, like separate areas and timing of meals for each staff [3]. NICE (2020) guidelines from the UK suggests that those $\mathrm{HCW}$ who have to self-isolate themselves can help with remote tele consultations, contouring, and communications [4]. A system-wide "COVID Huddle" video-conference daily was suggested at John Hopkins [5]. Italian experience proposed borrowing staff from other facilities and to call back retired staff, to continue services [6].

Policy that can be implemented:

1. Formation of a "COVID-19 emergency team" including the head of the department for dynamic monitoring of policy, addressal of staff problems, PPE availability and if needed, change in policies.

2. Meetings at least twice a week (by video conferencing) with all working staff for feed-back, functioning and safety.

3. If adequate strength available, all staff may be divided into 3 teams- Team A, Team B and Team C. Team A and B can work by rotation for a minimum of
7 days each. When Team A is working, Team B works from home, assisting in remote conference patient visits, remote contouring and planning. TeamC are deployed in essential COVID-19 duties.

4. Remote access licences for the treatment planning systems need to be worked out with the vendors.

5. Divide RT area into different zones based on contamination levels. The respective areas are to be disinfected accordingly. Efforts must be taken to ensure minimal intermixing of staff posted in respective areas.

6. Discourage staff borrowing as the staff strength is already compromised and the legal issues involved. Take all measures to protect the staff from infection to continue services.

\section{Staff Education}

Published guidelines: Detailed guideline has been provided by the WHO (2020) which covers hand washing, respiratory hygiene, physical distancing, self quarantine for those with travel history, which must be practiced by all, including a HCW. Guidelines on rational use of PPE has been published by the Ministry of Health and Family Welfare (MOHFW) Govt. of India [7].

Policy that can be implemented:

1. Demonstrations of proper PPE usage and disposal, behavioral changes to be adopted including physical distancing, hand hygiene and respiratory etiquettes both while dealing with a patient and outside the hospital, must be given to all staff, preferably by an expert from the infection control team.

2 . They can be trained by the medium of presentations, mock drills and telemedicine.

3. Emergency preparedness and contingency plan should be discussed

4. Physical distancing among the HCWs and patients has to be followed strictly at all times including tea times and lunch breaks.

\section{Screening}

Published guidelines: Very stringent screening of patients was done in Wuhan. Masks were compulsory. Patient and attender would first go through a temperature checkpoint. At John Hopkins also an intensive screening was followed for respiratory symptoms which if present, patients were assigned to a specific primary health care. [5]. A policy of bracelets for screened patients was followed by Italian centers [8]. In certain centers in Washington, thermal scanners were installed in entrances [9]. In Iranian centers, health workers were required to change clothes at entry and exit [10]. In Taiwan, if the patient has travelled to a hotspot zone in the last 2 weeks, then they were prohibited from entering hospitals till completing a 14-day quarantine [11].

Policy that can be implemented:

1. A queue with physical distancing (marked as circles for standing) should be drawn before all entryways. Patient alone or maximum one caregiver be allowed.

2 . The wearing of masks must be compulsory for both the patient and the caregiver. Hand wash and sanitization for every person entering and exiting the building must 
be made compulsory.

3. A self-declaration form which includes travel history, contact history, respiratory symptoms and fever is to be filled by patient and caregivers. A declaration that they may contract infection during treatment can be signed. Online forms that can be filled and submitted by the patient from their mobile is preferred to decrease the chance of infection associated with paper forms. [appendix A1] \& (https://form.jotform. com/201074359399463).

4. Thermal scanning with a hand held non touch thermometer is advised..

5. A separate entry and exit for patients may be provided.

\section{Waiting Area}

Published guidelines: Italian centers modified the waiting areas with each chair being spaced more than 1 metre and articles like magazines and newspapers removed from the waiting area [8]. The Iranian experience highlighted separate isolated waiting areas for those who had symptoms of cough, fever or unexpected malaise or myalgia. If the number of people had exceeded the capacity of the waiting room, they were advised to sit in their cars or areas outside the hospital [10]. NICE guidelines have highlighted the importance of scheduling a patient carefully, such that the patient does not have to wait for a prolonged time unnecessarily [4].

Policy that can be implemented:

1. Decrease the number of chairs available in the waiting area, spacing at least one meter between chairs.

2. Pictorial depiction of personal etiquettes like handwashing, respiratory hygiene and physical distancing can be displayed on the walls of the waiting area.

3. Careful scheduling of time-slots for treatment/ simulation is mandatory to minimize undue delay for treatment and decrease the time spent in the waiting area.

4. Waiting area has to be sanitized frequently

\section{Patient Selection}

Published guidelines: The UK NICE (2020) have given comprehensive guidelines for selection of patients for radio-therapy and divided patients into 5 groups [4]. The highest priority was given to the rapidly growing tumors like that of head and neck, cervix, and post operative with residual disease; least priority given to prostate, and hormone positive breast cancers. The RADS (Remote, Avoid, Defer, Shorten) principle had been advocated. John Hopkins University divided patients into different levels from 1 to 3 . Level 1 are those that would continue radiation. This includes those who were already on radiotherapy and those who require urgent radiotherapy in a palliative setting or rapidly proliferating tumor in curative setting. Level 2 includes routine sites that require radiotherapy. Level 3 included those cases where RT could be delayed or omitted altogether [5]. For Head and neck cancers, concurrent chemotherapy can be omitted for patients 70 years or older with co-morbidities, such as diabetes and cardiovascular diseases. Chinese centers did not prioritize as less patients visited the facility due to lock down.

Policy that can be implemented:

1. Patients in whom to avoid radiotherapy altogether:

a. Hormone positive, elderly women with carcinoma breast post breast conservation surgery and started on hormonal therapy.

b. Palliative radiotherapy in patients with ECOG of 3 or 4 (very unlikely to benefit).

c. Defaulted patients due to personal, logistic or post-operative complications, who have presented for post-operative radiotherapy where the window of benefit has passed.

2. Patients in whom to delay radiotherapy:

a. Carcinoma prostate, kept on Androgen deprivation therapy (ADT)

b. Carcinoma Breast: If adjuvant radiotherapy is indicated, a delay of up to 6 to 7 months after surgery and 1 month after adjuvant chemo is acceptable. For hormone positive tumors, hormone therapy can be started and a slightly longer delay may be acceptable.

c. Head and Neck Cancers: In patients started on neoadjuvant chemotherapy, with good response and tolerance, RT can be delayed with 2 or more additional cycles of chemotherapy. Post op radiotherapy in salivary gland tumors may be prolonged.

d. GI cancers: Adjuvant radiotherapy in carcinoma of rectum, stomach, pancreas and gallbladder may be delayed and adjuvant chemotherapy can be started.

e. Brain tumors: Grade 2 tumors post complete excision may be advised for delayed radiotherapy and follow up. For those with incomplete excision or grade 3 tumors and certain elderly patients with grade 4 gliomas may be started on Temozolomide for 2 to 3 cycles to delay radiotherapy. Radiotherapy maybe differed for benign tumors like meningiomas and arteriovenous malformations.

f. Sarcomas: Most low or intermediate grade sarcomas may be considered for delayed radiotherapy. Ewing's sarcoma and Rhabdomyosarcoma can continue chemotherapy till the control of the pandemic.

g. Carcinoma Lung: Those receiving neoadjuvant chemotherapy, with good response, may be continued for a few more cycles, before start of radiotherapy. Prophylactic cranial irradiation in small cell lung cancer may be delayed or avoided.

h. Palliative radiotherapy: For patients with no acute symptoms that need palliation, RT can be delayed. Also for bone metastasis in non-weight bearing sites with adequate control of pain with analgesics, RT may be delayed.

3. Patients in whom radiotherapy must be given:

a. Patients already on radiotherapy should continue radiation. They can be considered for modification to a hypofractionated regimen.

b. Rapidly proliferating tumor (head and neck, cervix, anal canal, esophagus, inoperable vulva and vagina, post operative with residual disease in fast growing tumors, young grade 4 gliomas).

c. Urgent palliative radiotherapy which gives good symptomatic relief, such as malignant spinal compression, 
superior vena cava syndrome or bony pain.

d. Adjuvant radiotherapy may be given with low priority if local recurrence risk is more than $20 \%$ at 10 years.

\section{Radiotherapy Fractionation}

Published guidelines: The most established fact is that "shorter the treatment, the better", which highlights the importance of hypofractionation [12-13]. For breast cancer, the guidelines for the pandemic have evolved rapidly. Hypofractionation with a dose of 40 Gy in 15 fraction and 42.5 Gy in 16 fraction has been the most accepted regimens. Extreme hypo fractionation has also been adopted in many centers with a dose of $26 \mathrm{~Gy}$ in 5 fraction. Boost has been avoided in those less than 40 years of age. External beam Partial breast irradiation of $40 \mathrm{~Gy}$ in 10 fraction or 30Gy in 5 fractions has also been adopted [14]. For carcinoma of rectum short course radiotherapy is preferred. In certain centers, organ preservation is also advocated with surgery reserved as salvage for recurrence [15]. In head and neck, definitive chemo-radiation should be given along with simultaneous integrated boost (SIB) techniques in the standard (5 fractions per week) or accelerated schedule (6 fractions per week) in order to achieve a 1-week reduction compared to sequential technique [16]. RCR suggested to consider 65 Gy in 30 fractions or $55 \mathrm{~Gy}$ in 20 fractions over standard $70 \mathrm{GY}$ in 35 fractions with limiting chemotherapy over 60 year.

Policy that can be implemented:

1. Carcinoma Breast: Extreme hypofractionated regimens like 28-30 Gy in 5 weekly fractions, (FAST protocol) or 26 Gy/5 Fractions/ 1 week (FAST forward) may be given preference if radiotherapy is planned. If these are not possible, $40 \mathrm{~Gy} / 15 \mathrm{fr} / 3$ weeks may be used instead of conventional regimens. Avoid boost dose for all. If at all compulsory, go for a simultaneous integrated or $10 \mathrm{~Gy} / 4 \mathrm{fr} / 4$ days sequential boost (for $<40$ years only). Encourage external beam accelerated partial breast irradiation (APBI) protocol of $30 \mathrm{~Gy} / 5$ Fractions in suitable patients.

2. Head and Neck Cancers: A dose of $65 \mathrm{~Gy} / 30 \mathrm{fr} / 6$ weeks by SIB-IMRT technique is advocated instead of 70 Gy/ 35 fr/ 7 weeks. A dose of 60-64Gy in 30-32 fractions is used in postop settings. Concurrent chemotherapy to be avoided above 60 years.

3. Carcinoma Cervix: 50 Gy in 25 fractions followed by a 3D IMRT/ SBRT boost of 10-16 Gy (If brachytherapy not possible). Concurrent chemotherapy to be given.

4. Carcinoma prostate: It is better to delay prostate radiotherapy at this time. If planned they can receive $60 \mathrm{~Gy}$ in 20 fractions over 4 weeks. SBRT protocols may be given priority over moderate hypofractionation in localized disease, if the logistics for planning and delivery permit.

5. GBM: $25 \mathrm{~Gy} / 5 \mathrm{Fr} / 1$ week may be given if RT is considered.

6. GI malignancies: There is no role of hypofractionation in these settings and are treated in the conventional dose fractionations of 45-54Gy in 1.8-2Gy per fraction, except in rectum, where preop short course
RT of 25 Gy in 5 fractions can be given.

7. Carcinoma Lung: Conventional fractionation will be followed up to a dose of 60Gy in 30 fractions over 6 weeks. Prophylactic cranial irradiation may be avoided or delayed.

8. Palliative radiotherapy: 8 Gy in single fraction should be advocated for spinal cord compression, impending fractures and severe bone pains. For SVCO one may consider 8.5 Gy weekly for two fractions. Brain metastases may be considered for whole brain radiotherapy of $20 \mathrm{~Gy}$ in 5 fractions.

\section{Treatment Planning and Execution}

Published guidelines: Italian experience described how CT simulation of patients was dispersed at different times of the day, to decrease waiting outside CT-simulation room [8]. Most published guidelines advocate treatment as less complicated as possible. For those patients who have started treatment with active breath coordinator (ABC) can be completed using the same unless the patient becomes COVID-19 positive. It is advisable not to start any new patients with $\mathrm{ABC}$ during this time [17]. Experience from Switzerland highlights the need for making less complicated plans, and limits the use of complicated IMRT, SBRT and SRS only if necessary, as this would avoid the need for patient specific quality assurance by a medical physicist [18].

Policy that can be implemented:

1. 2 Dimensional planning in telecobalt or a linear accelerator should be preferred if possible, as the treatment can be started on the same day and less manipulation on the machine during setup. Hypofractionated treatments may be given with conformal and image guidance techniques.

2. CT-based planning may be done only for carefully selected patients. Special care must be taken while placing fiducials, giving oral contrast (ca esophagus), intravenous contrast, specially after cross checking past history of allergy to avoid unnecessary need for resuscitation in case of anaphylaxis.

3. Licences for remote planning and contouring may be obtained from the vendors.

4. Each planning, treatment and set-up should be followed by appropriate disinfecting of the surface of the couches, immobilization devices, other radiotherapy accessories like head rests, knee rests, breast boards, baseplates thermoplastic masks, etc with appropriate disinfectants like 1\% sodium hypochlorite. Care should be taken to frequently disinfect the mouse and keyboards.

5. Thermoplastic casts of each patient should be kept separately.

6. Skin marking is preferred for all palliative patients as this helps in avoiding unnecessary contact with staff for making thermoplastic sheets and avoids need for sanitization of baseplates and casts.

7. History pertaining to symptoms of COVID should be asked every day by the technical staff before taking the patient for treatment. Any COVID-19 positive/suspected patient should have their treatment stopped and restarted only after 2 negative results. 
8. The total number of patients on the machine needs to be restricted and spread out with intervals to avoid crowding and to give time for sanitisation in between.

9. Avoid complicated treatment planning, like stereotactic body radiotherapy (SBRT), SRS as it can increase the risk of transmission (longer and multiple interactions, longer treatment setups and added radiotherapy accessories).

10. Avoid using ABC.

\section{Review of Patients on Treatment}

Published guidelines: Although specific guidelines have not been published specifically for reviewing patients on radiotherapy, it has been encouraged to have maximum review and follow up through tele-communications, WhatsApp or video-conferencing.

Policy that can be implemented:

1. Reviewing of patients on treatment should be as minimal as possible, and can be encouraged by tele-communication or video conferencing platforms like whatsapp, preferably by HCW working from home.

2. For physical review, a minimum of 1 to 2 meter distance has to be maintained between patients presenting with such symptoms.

3. Patients coming for review may be advised to come alone without any attendant, unless the patient is a child, sick or has difficulty in comprehension.

4. Routine blood investigation may be avoided unless absolutely necessary.

\section{Brachytherapy}

Published guidelines: The most important one is the ABS (American Brachytherapy Society) (2020) guideline, which gives strict instruction to continue the treatment of carcinoma cervix in patients whose treatment has already started and high risk carcinoma prostate planned for EBRT and Brachytherapy boost [19]. Endometrial cancer requiring brachytherapy may be delayed till 12 weeks, however 9 weeks is recommended. Experts from Switzerland had anticipated the shortage of anesthetists and recommended sedoanalgesia (sedation plus local anesthesia). They also increased EBRT dose to avoid brachytherapy. RCR has proposed adapting the treatment pathway to deliver 2 to 3 fractions per insertion with 6 hours gap.

Policy that can be implemented:

1. In cervical cancers, all the three fractions can be delivered with a single applicator insertion keeping the patient admitted with the radiotherapy delivered 6 hours apart, instead of weekly fractions. Alternately 9 Gy x 2 weekly (PGIMER regimen) may be given.

2. Insertions may be performed under sedation rather than spinal or general anaesthesia.

3 . Vault brachytherapy may be continued.

4. Endometrial cancer brachytherapy in intermediate risk should be avoided.

5. Interstitial implants to be avoided as it will require anaesthesia, which may not be available.

6. Replace Interstitial implants with external beam radiotherapy (EBRT).
7. Brachytherapy for prostate cancer is not justified in this pandemic and may be avoided.

8. Head and neck brachytherapy, Intraluminal brachytherapy for esophagus and lungs are strictly prohibited.

9. Breast brachytherapy may be avoided and replaced with EBRT.

\section{Inpatient Admissions}

Published guidelines:

There is paucity of data regarding criteria for admissions, although the requirement would be low. Chinese had a very strict policy regarding admission with every patient and attender being screened with CT chest, and all symptomatic patients being tested for COVID 19, and admitting only if negative [2]. Italian experience in pediatric oncology suggests a single parent accompanying the child, a single child in recovery after GA and sterilization of all toys on a daily basis [20].

Policy that can be implemented:

1. All patients requiring admission must strictly be screened, with a very low threshold for COVID-19 testing.

2 . The number of beds in a cubicle may be restricted to follow strict physical distancing guidelines.

3. For those requiring admissions like radiotherapy under GA and very sick patients like SVCO syndrome, symptomatic brain metastasis etc, all admissions are to be considered only after thorough screening of patients and attenders along with COVID-19 testing for any suspicious patients. A compulsory CT scan may not be feasible or justified in view of the radiation risk and contamination.

4. All admitted patients and attenders should be given proper health education, encouraged to wear face masks and have their hands sanitized periodically.

\section{Follow up Policy}

Published guidelines: All published guidelines strongly recommend tele-communication and video consultations. Patients with prior appointments may be contacted to postpone them.

Policy that can be implemented:

1. Teleconferencing platforms like Whatsapp can be used as the primary modality of communication.

2. Patients should be educated regarding the signs and symptoms of recurrence or disease progression.

3. Follow up investigations like Whole Body PET-CT, MRI, CECT, DEXA scans, 2D ECHO, blood investigations etc may be postponed.

4. Remedies for radiation reactions and adjuvant treatments like hormonal therapy may be advised over teleconference.

5. The frequency of followup may be decreased.

\section{Resident Training}

Published guidelines: United States groups did focus on resident training. All lectures and didactic activities had been converted to videoconference.

Policy that can be implemented:

1. Academic training should be started through videoconferences. 
2. Residents may be encouraged to use their spare time judiciously when 'working from home' to focus on studies and writing research grants and manuscripts for publications.

3. Assessments may be postponed or may be conducted carefully following norms.

\section{Radiotherapy Treatment of Covid-19 Suspected or Positive Cancer Patients}

Published guidelines: According to RCR UK (2020), COVID-19 suspected patients should follow standard norms as for other COVID-19 patients with an oncology team at close reach [21]. If a patient is infected in between treatment, further treatment should be interrupted till cured of infection and treatment restarted after gap correction. As per John Hopkins, suspected or positive patients should only be treated if they meet the criteria for urgent RT, and they are the patients with rapidly progressive curable disease, symptomatic metastatic disease with life expectancy of at least 3-6 months in whom alternative treatment is not possible. These treatments should be done at the end of the day, with appropriate PPE, entry and exit from low volume entrance and following all infection prevention control measures [5].

Policy that can be implemented:

Due to lack of isolated RT facilities and keeping in mind the risk of contagion to other fellow departments, suspected or positive cases should not be treated until they are cured.

All decisions should be taken tailoring to the specific needs of the patients based on the present situation of the pandemic and available resources at the center. One may have to accept the compromise in the overall survival of these patients in these uncertain times.

\section{References}

1. Dai M, Liu D, Liu M, Zhou F, Li G, Chen Z, Zhang Z, You H, Wu M, Zheng Q, Xiong Y, Xiong H, Wang C, Chen C, Xiong F, Zhang Y, Peng Y, Ge S, Zhen B, Yu T, Wang L, Wang H, Liu Y, Chen Y, Mei J, Gao X, Li Z, Gan L, He C, Li Z, Shi Y, Qi Y, Yang J, Tenen DG, Chai L, Mucci LA, Santillana M, Cai H. Patients with cancer appear more vulnerable to SARS-COV-2: a multi-center study during the COVID-19 outbreak. Cancer Discovery. 202004 28;:CD-20-0422. https://doi.org/10.1158/2159-8290.cd-20-0422

2. Wei W, Zheng D, Lei Y, Wu S, Verma V, Liu Y, Wei X, Bi J, Hu D, Han G. Radiotherapy workflow and protection procedures during the Coronavirus Disease 2019 (COVID-19) outbreak: Experience of the Hubei Cancer Hospital in Wuhan, China. Radiotherapy and Oncology. 2020 07;148:203-210. https:// doi.org/10.1016/j.radonc.2020.03.029

3. Tey J, Ho S, Choo BA, Ho F, Yap SP, Tuan JK, Leong CN, Cheo T, Sommat K, Wang ML. Navigating the challenges of the COVID-19 outbreak: perspectives from the radiation oncology service in singapore. Radiotherapy and Oncology. 2020 03; https://doi.org/10.1016/j.radonc.2020.03.030

4. NICE guideline: COVID-19 rapid guideline: delivery of radiotherapy NICE guideline Published: 28 March 2020 www.nice.org.uk/guidance/ng162. Accessed on16 April 2020 ..

5. Wright JL, Alcorn SR, McNutt T, Han-Oh S, Gonzalez R, Lin
L, Anderson R, Wieworka J, Deville C, Ladra M, Meyer J, Hales R, Voong R, Narang A, Vogel J, Asrari F, Kiess A, Song D, Tran P, Kleinberg L, Redmond K, Tsien C, Quon H, Page B, Croog V, Walker A, Greco S, Laiho M, Viswanathan A. An Integrated Program in a Pandemic: Johns Hopkins Radiation Oncology Department. Advances in Radiation Oncology. 2020 04;. https://doi.org/10.1016/j.adro.2020.03.014

6. Filippi AR, Russi E, Magrini SM, Corvò R. Letter from Italy: First practical indications for radiation therapy departments during COVID-19 outbreak. International Journal of Radiation Oncology*Biology*Physics. 2020 03;. https:// doi.org/10.1016/j.ijrobp.2020.03.007

7. WHO interim guidance 2020. CC BY-NC-SA 3.0 IGO. Corona Virus ( COVID-19) outbreak: rights, roles and responsibilities of health workers including key considerations for occupational safety and health. Accessed on16 April 2020..

8. Krengli M, Ferrara E, Mastroleo F, Brambilla M, Ricardi U. Running a Radiation Oncology Department at the Time of Coronavirus: An Italian Experience. Advances in Radiation Oncology. 2020 03;. https://doi.org/10.1016/j. adro.2020.03.003

9. Dinh TT, Halasz LM, Ford E, Rengan R. Radiation Therapy in King County, Washington During the COVID-19 Pandemic: Balancing Patient Care, Transmission Mitigation, and Resident Training. Advances in Radiation Oncology. 2020 03;. https://doi.org/10.1016/j.adro.2020.03.007

10. Samiee S, Hadjilooei F, Alamolhoda M, Akhlaghpoor S. New Policy and Regulations for a Radiology-Oncology Center During the COVID-19 Outbreak in Tehran, Iran. Advances in Radiation Oncology. 2020 04; https://doi.org/10.1016/j. adro.2020.04.005

11. Chen Y, Hsu F, Tsai CJ, Cheng JC. Efforts to Reduce the Impact of Coronavirus Disease 2019 Outbreak on Radiation Oncology in Taiwan. Advances in Radiation Oncology. 2020 04;. https://doi.org/10.1016/j.adro.2020.03.005

12. Hanna TP, Evans GA, Booth CM. Cancer, COVID-19 and the precautionary principle: prioritizing treatment during a global pandemic. Nature Reviews Clinical Oncology. 2020 04 02;17(5):268-270. https://doi.org/10.1038/s41571-0200362-6

13. Zaorsky NG, Yu JB, McBride SM, Dess RT, Jackson WC, Mahal BA, Chen R, Choudhury A, Henry A, Syndikus I, Mitin T, Tree A, Kishan AU, Spratt DE. Prostate Cancer Radiation Therapy Recommendations in Response to COVID-19. Advances in Radiation Oncology. 2020 04;. https://doi.org/10.1016/j.adro.2020.03.010

14. Braunstein LZ, Gillespie EF, Hong L, Xu A, Bakhoum SF, Cuaron J, Mueller B, McCormick B, Cahlon O, Powell S, Khan AJ. Breast Radiation Therapy Under COVID-19 Pandemic Resource Constraints-Approaches to Defer or Shorten Treat-ment From a Comprehensive Cancer Center in the United States. Advances in Radiation Oncology. 2020 04;. https://doi.org/10.1016/j.adro.2020.03.013

15. Marijnen C, Peters F, Rödel C, Bujko K, Haustermans K, Fokas E, Glynne-Jones R, Valentini V, Spindler K, Guren M, Maingon P, Calvo F, Pares O, Glimelius B, SebagMontefiore D. International expert consensus statement regarding radiotherapy treatment options for rectal cancer during the COVID-19 pandemic. Radiotherapy and Oncology. 2020 07;148:213-215. https://doi.org/10.1016/j. radonc.2020.03.039

16. De Felice F, Polimeni A, Tombolini V. The impact of Coronavirus (COVID-19) on head and neck cancer patients' care. Radiotherapy and Oncology. 2020 06;147:84-85. https://doi.org/10.1016/j.radonc.2020.03.020 
17. Song AJ, Manukian G, Taylor AK, Anne PR, Simone NL. Concerns for Active Breathing Control (ABC) With Breast Cancer in the Era of COVID-19: Maximizing Infection Control While Minimizing Heart Dose. Advances in Radiation Oncology. 2020 04;. https://doi.org/10.1016/j. adro.2020.03.009

18. Papachristofilou A, Finazzi T, Kohler G, Dott C, Zimmermann F. Contingency Plans in a Radiation Oncology Department Amid the 2019-nCoV Outbreak in Swit-zerland. Advances in Radiation Oncology. 2020 03; https://doi.org/10.1016/j. adro.2020.03.012

19. ABS releases statement on brachytherapy services during COVID-19 pandem-ic. Appl Rad Oncol. https:// appliedradiationoncology.com/articles/abs-releasesstatement-on-brachytherapy-services-during-covid-19pandemic Accessed 16 April 2020.

20. Mascarin M, Coassin E, Provenzi M, Franchin G, Mariotto A. How to Reorganize Children's Access to Radiation Therapy in the Era of COVID-19, to Protect Them and Elderly Patients. Advances in Radiation Oncology. 2020 04;. https://doi.org/10.1016/j.adro.2020.04.002

21. RCR-UK: https://www.rcr.ac.uk/sites/default/files/cancertreatment-gaps-covid19.pdf. Accessed 16 April 2020..

\section{Qब}

This work is licensed under a Creative Commons AttributionNon Commercial 4.0 International License. 